\title{
Characterisation of an Electronic Booking System in the National Health Service: A Semiotic Perspective
}

\author{
Sanjayan Solangasenathirajan ${ }^{(凶)}$ and Keiichi Nakata \\ Henley Business School, Informatics Research Centre, \\ University of Reading, Reading, UK \\ s. solangasenathirajan@pgr.reading.ac.uk, \\ k.nakata@henley.ac.uk
}

\begin{abstract}
The NHS in England comprises multiple organisations, systems and actors working in co-ordination to deliver healthcare to the population. Transfers of care from primary care organisations is facilitated by an electronic booking system that is provided nationally, and utilised regionally and locally. The success of such system is dependent on the co-operation of actors and organisations in the health economy in affecting its impact and usefulness. Such a system can be seen as an 'artefact' where multiple worlds collide. The theory of boundary objects is approached from a critical realist paradigm to conceptualise the role of the focal system. Organisational semiotics is used to provide an analytical method to interpret the behaviours and perceptions surrounding the artefact. Understanding of these factors provides recognition of the tensions in adoption of the system. Implementation and expert use of systems considering these invisible factors can benefit from greater intellectual buy in and value co-creation.
\end{abstract}

Keywords: Critical realism - Organisational semiotics - Boundary objects • Computer supported co-operative work $\cdot$ Electronic referrals $\cdot$ National Health Service

\section{Introduction}

Interoperability of healthcare systems at the macro, meso and micro levels is high on the research agenda and in practice within the National Health Service (NHS) of England. Much of the effort and activity has been focused on the technical integration of systems through core services, linking of datasets, definitions and technical interfaces [1]. Even though, the NHS struggles to achieve effective integration and interoperation locally, within or across organisations in the local health community, or nationally across regions and geographies. Lack of social collaboration in the technical design, development and implementation of systems could be seen as a barrier to healthcare systems interoperation.

An understanding of the interfaces, and the behaviours and perceptions of the stakeholders who take an interactive role within and around these collaborative systems is required, to understand the socio-technical aspects of co-ordinated system utilisation 
and the variance of interpretations and barriers to perceived benefits, which result in negative effects. This paper seeks to characterise the role of such a system in its society and the assumed responsibilities of those actors within it. Through the analysis of the human information context it is postulated that a greater understanding of the intrinsic nature is developed to establish the requirements from co-coordinative domains.

\section{NHS Electronic Booking System}

A number of core national information systems are currently in use by the NHS in England, of which one facilitates the electronic transmission of referral and appointments booking information across NHS organisations nationally. Its fundamental use is to refer a patient from a primary care organisation to a secondary care organisation, and to support the transfer of clinical information, service selection and appointments booking. This information system is based on core clinical Directory of Services, from which referrers and patients can choose and book an appointment date and time of their preference, in a clinical service of their choice. The system is populated by clinical service definitions from providers of NHS services and integrates with the patient administration systems, so that appointment slots can be polled and made available to the referrals and appointments system. Additionally, referrers can initiate the creation of a referral and attach content from within their GP clinical system. Access is based on user roles and governed by Role Based Access Controls on smartcards used to access the system. Users of the system have referring, service provider, commissioning and further supporting roles with the appropriate functionality. The information within this system can be utilised to understand 'future outpatient' activity and the demands and trends of populations on secondary care clinical services.

This information system can be classified as one which acts as a focal point for the interoperation of a number of clinical information systems and co-operative work [2] of a variety of stakeholders in the formal and informal system [3]. The electronic referral and appointments booking system can be treated as an 'artefact' that sits between and forms many 'boundaries' with organisations and their actors. The actors in these organisations take on a number of roles in which they play a part in the function of the system. These use cases can be technical, non-technical, facilitating or beneficiary.

\subsection{Design Intentions}

The electronic referrals and appointments booking system is utilised in the NHS as part of a core provision of national information services. The core principle was to enable direct access and booking from primary care to secondary care. More specifically: to support patient choice of clinical service and appointment date/time; to support clinical Service Providers to triage and accept referrals that are clinically appropriate only once the patient has been provided an appointment; to facilitate advice and guidance in supporting a referral decision thereby reducing first outpatient appointments through activity diversion and reducing waiting times. Up to date and accurate definition and publication, provides a shop window for Service Providers, for referrers navigating and identifying their services. 


\subsection{System Characterisation}

Figure 1 illustrates the system as a mediating artefact in a network of stakeholders and organisations (indicated the first level), and systems/functions within that organisation.

The system is at the core of interfacing organisations working to co-ordinate activity to facilitate the process of patients 'choosing' and 'booking' an appointment within a Provider service of their choice. Clinical Commissioning Groups play a beneficiary role in the use of management information, delivery of policy and governance over national performance and quality targets. General Practice and the Service Providers play technical and non-technical roles, with the end users acting with the system and management stakeholders playing non-technical roles in the informal system. The Commissioning Support Unit plays a facilitating role in operational support to General Practice, strategic enablement to the Clinical Commissioning Groups and an interfacing role to support and bring awareness of the electronic booking system. With view of the system as an artefact, differences in local and regional norms cause variation in these multiple domains. This leads to a great degree of permutation in use, from design intention.

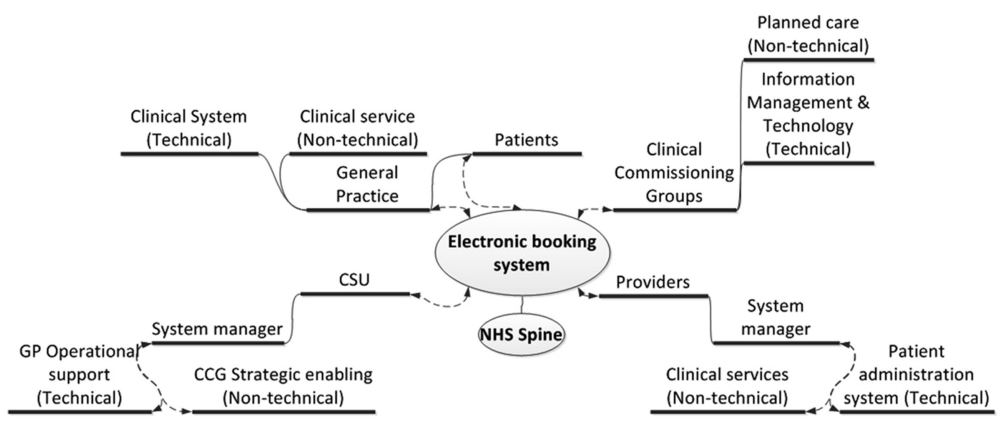

Fig. 1. Stakeholders of the NHS electronic booking system

\section{Boundary Objects}

The national electronic referrals and appointments booking system can be treated as an artefact, classified as a boundary object [4] in-use, which is comprised of systems acting as repositories and ideal-type concepts provided by definitions. These definitions could be formalised or interpreted to convey different meaning based on the context in which it is being used. A boundary object may be performed differently across multiple sites, times, practices and participants, with varying effects [5]. Boundary objects perform a brokering role [6] involving translation, coordination and alignment between the interfaces and perspectives of specific Communities of Practice [7]. Boundary objects facilitate cooperation between intersecting communities by maintaining a common identity while satisfying the informational needs of each $[4,8]$ and provide a locus for communication, conflict, and coordination [9] and for actors with diverse goals to work together [10]. 
The interaction between a user and a system is a conversation in a vocabulary and language determined by the input the system is able to accept and process. Many systems (e.g., help systems, documentation systems) are oriented toward the system rather than toward the user: information is structured around a description of the system, not necessarily around an analysis of the problems users address when using the system [11]. As the electronic booking system is dependent on the co-ordination and collaboration of multiple stakeholders, variation in utilisation and system failure can be caused by neglect by one of the communities it is intended to bridge [12]. Boundary objects require a joint field and incorporation into local practice to become boundary objects-in-use [13] and have material aspects that afford or constrain boundary-spanning practices [14]. They enable knowledge transfer and the translation of meaning and interests, but their boundary-spanning effectiveness or role may vary across settings or time [4, 15, 16].

Studies have shown that artefacts can fail as boundary objects when they do not fully or rightfully capture multiple meanings and perspectives [17]. This interpretive flexibility leads to a misalignment between the IT structure and work process needs and arrangements, and the dynamic between ill-structured and more tailored uses of the objects [18].

Even though the current system has been in operation for approximately 10 years, the utilisation nationally varies to a great extent. Issues from system conception still manifest themselves as ongoing issues to date [19], thus engagement from end users and managers in some places has remained poor and perceptions formed and embedded through bad experience, relationships or interactions [20]. Boundary objects provide a bridging function but additionally need supporting awareness practices [21]. The system is characterised as a boundary object so as to facilitate the conceptualisation of the multiple connected domains, and analysis of their contributory elements.

In this paper we study boundary objects through a semiotic lens. This is appropriate as, for example, the integrative semiotic framework [22] provides a method of analysis of the environmental and social context so that a greater understanding of user requirements can be developed in contribution to gaining intellectual buy in [23]. Moreover, the semantic and pragmatic levels of the semiotic ladder [24] provide aspects of assessment into the characteristics of the boundary object. The pragmatics provide an insight into the communicated intentions of the boundary object and how this influences actors. The semantics looks to uncover individual and the wider collective meaning derived from various signifying factors, in the way so to uncover further issues in the informal system, and contributory factors in domain norms resulting in impedance of innovation and system failures. Subsequently to reveal the gaps between system design, governance mechanisms and situated use.

\section{A Semiotic Analysis of NHS Electronic Booking System as Boundary Object}

The study was approached by conducting semi-structured interviews to capture the semantic and pragmatic views of the system from its users and stakeholders and to provide a basis for cross examination of these findings with the design intention of the 
system. This semiotic analysis is to determine the success of the system in fulfilling its design intentions and in highlighting and understanding the causes for failure, where it is not used as intended. Ethnography is used to support or negate this analysis in observing stakeholders in use or co-ordination around the focal system, to provide a basis for highlighting and commenting on the successes and failures from the semiotic perspective.

\subsection{Signification}

Each has a part to play in the design intention of the system, in that which is signified and that which is interpreted by the various stakeholders. The system as a boundary object artefact signifies its design intentions. The expected use of the system is interpreted and observed by the various actors in accordance with their existing tasks, practice and position, as illustrated in Fig. 2.

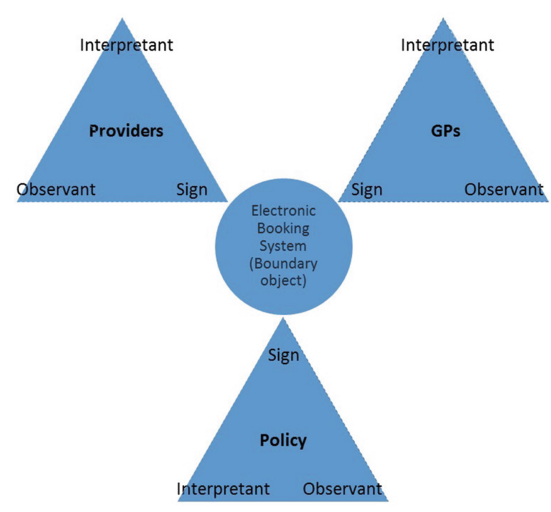

Fig. 2. Signification of system as boundary object

Superficially, the system is observed as merely providing a means to transfer referral information from one organisation to another. However, it serves different meanings and intensions for different stakeholders, e.g., facilitating the strategic objectives of patient choice, waiting time targets, and activity diversion. This is primarily influenced by local governance within the informal system.

\subsection{Semiotic Analysis}

The semantic and pragmatic perspectives of each system role, as adopted by stakeholders in the information system has been summarized in Table 1.

The system process models the series of tasks that occur for end user interacting with the system from its design intentions. This is described from the GP perspective as illustrated in Fig. 3. In practice, these tasks can be carried out by different users, such as a Medical Secretary at differing time points. 
Table 1. Analysis of human information functions

\begin{tabular}{|c|c|c|}
\hline & Semantic & Pragmatic \\
\hline GP & $\begin{array}{l}\text { A comprehensive listing of locally and } \\
\text { nationally available services, } \\
\text { appointment dates/times. With ability } \\
\text { to seek advice from secondary care } \\
\text { colleagues }\end{array}$ & $\begin{array}{l}\text { Facilitates the clinically appropriate } \\
\text { identification of services for patient } \\
\text { referral and appointment booking. } \\
\text { Facilitates advice and guidance from } \\
\text { secondary care }\end{array}$ \\
\hline $\begin{array}{l}\text { Medical } \\
\text { secretary }\end{array}$ & $\begin{array}{l}\text { Comprehensive listing of locally and } \\
\text { nationally available services, } \\
\text { appointment dates/times }\end{array}$ & $\begin{array}{l}\text { Facilitates the clinically appropriate } \\
\text { identification of services for patient } \\
\text { referral, automated digital referral } \\
\text { attachments and appointment } \\
\text { booking }\end{array}$ \\
\hline $\begin{array}{l}\text { Practice } \\
\text { manager }\end{array}$ & $\begin{array}{l}\text { An electronic referral and appointments } \\
\text { system }\end{array}$ & Monitor and manage referral activity \\
\hline Consultant & $\begin{array}{l}\text { An electronic referral triaging and } \\
\text { advice and guidance provision } \\
\text { system }\end{array}$ & $\begin{array}{l}\text { Electronically review incoming } \\
\text { referrals and advice requests }\end{array}$ \\
\hline $\begin{array}{l}\text { Outpatients } \\
\text { manager }\end{array}$ & $\begin{array}{l}\text { An electronic appointments booking } \\
\text { system }\end{array}$ & $\begin{array}{l}\text { Directly book appointments and } \\
\text { manage slot availability }\end{array}$ \\
\hline $\begin{array}{l}\text { Service } \\
\text { manager }\end{array}$ & $\begin{array}{l}\text { Digitally define services within } \\
\text { specialty }\end{array}$ & Shop window of services \\
\hline
\end{tabular}

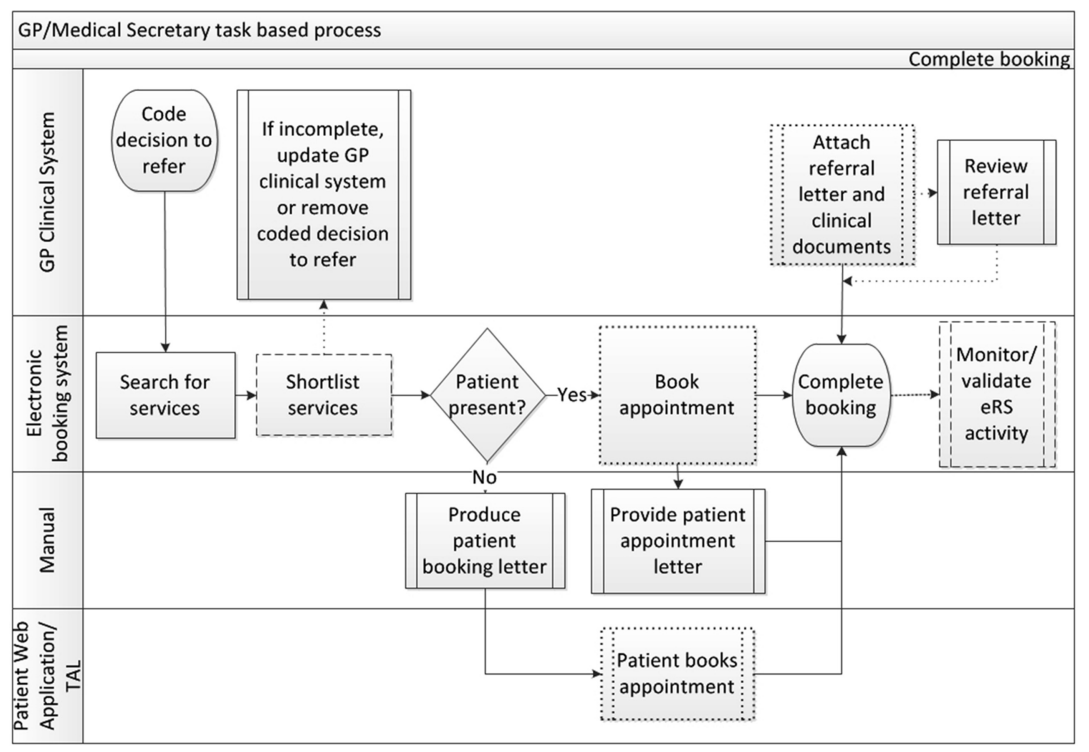

Fig. 3. Referrer booking 


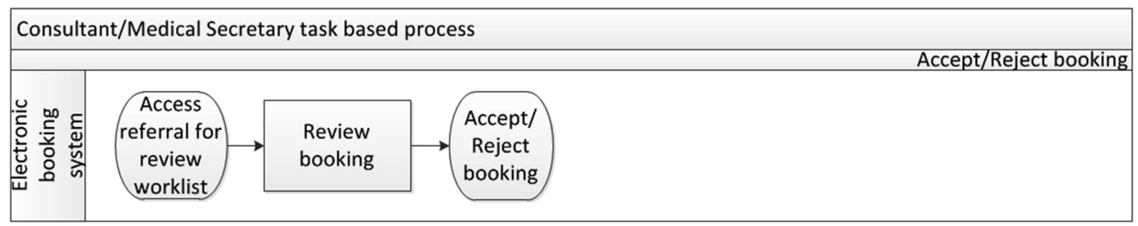

Fig. 4. Service Provider triaging

The 'book appointment' process releases any attached clinical information to the Service Provider, the referral attachments also need to have been attached into the electronic booking system. Due to the variety of clinical systems used in General Practice there are multiple methods by which the letter and clinical documents can be attached. Process variations and the timeliness of working through the process in Fig. 3 directly impact the efficacy of the process in Fig. 4.

In order for the 'review booking' process to take place the 'patient books appointment' and 'attach referral letter and clinical documents' sub processes in Fig. 3 need to have taken place in the time prior to the triaging of the booking. Timeliness of sub processes is key to the success of the system, without which the system fails. Where in Fig. 3 the processes in dashed lines are not carried out completely or routinely, adverse effects such as the GP clinical system recording that a booking has been created when in fact no booking has been created occur. Also, where bookings are rejected these are not picked up and therefore the patient has not progressed in receiving any care.

\subsection{Contextualisation of Semantic and Pragmatic Issues}

\subsubsection{Referrers}

GPs view the system as one which can support or impede the day to day clinical duty. Referring a patient is in some cases perceived as a task carried out by administrative staff. This transfer of information is in some cases supported by additional technologies, such as Dictaphones. Referral attachment content is produced by a variety of methods given the variety of up to 5 different GP clinical systems in use by practices, and within a GP clinical system, the particular way in which the patient details and referral prose is added. This leads to inconsistencies in the structure, quality of content impacting on the way that it is accessed and usefully perceived on the Service Provider side.

Where the shortlisting of services takes place there is inconsistency in the number of choices that are provided, and in some cases in merit of the choice discussion having already taken place externally to the system, only one choice would be shortlisted. The directory only publishes planned care services, so accommodates for a proportion of total GP referrals.

If the referral is not made prior to the patient leaving the practice following consultation, then the patient booking letter has to be posted to the patient so that their appointment can be booked. The cost incurred in generating and posting, negates any perceived benefit of the system. In some instances the appointment is booked without consultation with the patient and subsequently removing any requirement to generate 
the booking letter. This approach heavily relies on the Service Provider contacting the patient with appointment details, and negates intended system use around choice and ability for patients to manage their booking.

\subsubsection{Service Providers}

In Service Provider organisations the triaging consultant can directly access their referral through the system web interface and accept or reject that particular booking, as illustrated in Fig. 4. They are also enabled to access their 'advice and guidance' requests. Some instances describe where consultants never access the system and have the referrals passed to them in paper format, where they have been retrieved by administration staff. Service Providers do not always (have the capacity) to poll available clinic slots, so this can lead to subsequent manual processes where the patient has to be contacted and appointed manually.

In Service Provider organisations, there is also similar variation in each stage of the activity. Whereby some processes are carried out by clinical or administrative staff, in either centralised or distributed functions throughout the organisation.

Worklists determine the management and administration tasks that occur in the use of the system or in the management of events that have arisen from unintended circumstances. For example, if a referral is rejected as clinically inappropriate by the Service Provider, this then populates on a worklist in the GP practice. This should result in the patient being contacted by the GP practice about this cancellation. The adverse impacts of not monitoring this worklist are unforeseen by practices, and only experienced by the Service Provider and Patient, when a rejected appointment is attended.

In bridging all of this activity the system exhibits behaviour of a boundary object in use, whereby it facilitates an interfacing role. Each of the use cases contributes to a variety of interpretation and use of the artefact. Because of the local variances the sign conveys a different interpretation to observation, as intended. The overall use of the system changes over time and in instances can be influenced by external factors in the informal system. The stakeholder map in Fig. 1 highlights the external composition to a boundary object, in addition to that characterised within.

\section{Discussion}

The processes and protocols that take place locally are bound to the semantic, pragmatic and social norms of the stakeholders in that particular local instance. This is influenced and constrained by historical and current factors attributed to the signification of the focal system. The malleability of the system from its design intentions, as a boundary object has lent to a multitude of process variations, omissions and workarounds attributed to the ways in which it is interpreted and observed. This flexibility has increased its adoption in tune with local needs, but resulted in deviation from design intention. This also questions whether the design intention was correct, but also for its continuous evolution. Importantly, where beneficiaries as key influencers have least intellectual buy in, resulting interpretations of the system in that instance are subject to greater variation. In these cases, greater facilitation, interfacing and support is required to enable system design intention. 
Activity at the boundary is instrumental in shaping the semantics of the system, in setting expectations and resolving misunderstandings. The practical aspects of the system are addressed by collaborating with the group in representation of the system to minimize the emergence of group related issues. The wider social group works in isolation in each organisation, and within an organisation within departments. Internally, the system exhibits its own semiotic characteristics which impact on its wider social use. The greater role of beneficiaries and facilitators is under recognised in the implementation and utilisation of these boundary objects. A subsequent focus is required on the impact of the non-technical stakeholders, and in their use cases that affect the efficacy of the technical system design intentions and innovation governance [25].

\section{Conclusion}

A system classified as a boundary object signifies that which has varying interpretations and observations, impacted by its semiotic attributes. These affect the way in which the system fulfils its design intentions, and the subsequent effects of system malleability. Activity at the boundary and the stakeholders in the informal system are primary to influencing the instance of the system. The transition to a new open source system provides an opportunity to observe the participation in design. There is a need to bridge natural user protocols, to systems and governance protocols. Where co-design of system intentions takes place there is a reduction in its need for malleability, and co-creation of values based on learned incentives. Further work is required to investigate the factors affecting the efficacy and subsequent utilisation of the system and improving long term acceptance. In uncovering the factors that impact this we can seek to resolve the tensions between the normative values, forms of authority and order imposed by the system.

\section{References}

1. Clegg, C., Shepherd, C.: The biggest computer programme in the world...ever!: time for a change in mindset? J. Inf. Technol. 22(3), 212-221 (2007)

2. Schmidt, K., Bannon, L.: Taking CSCW seriously. Comput. Support. Coop. Work (CSCW) 1(1-2), 7-40 (1992)

3. Stamper, R.K.: Information in Business and Administrative Systems. Wiley, New York (1973)

4. Star, S.L., Griesemer, J.R.: Institutional ecology, "translations", and boundary objects: amateurs and professionals in Berkeley's Museum of Vertebrate Zoology, 1907-39. Soc. Stud. Sci. 19, 387-420 (1989)

5. Suchman, L.A.: Human-Machine Reconfigurations: Plans and Situated Actions. Cambridge University Press, Cambridge, New York (2007)

6. Fischer, G., Reeves, B.N.: Creating success models of cooperative problem solving. In: Baecker, R.M., et al. (eds.) Readings in Human-Computer Interaction: Toward the Year 2000, pp. 822-831 (1995)

7. Wenger, E.: Communities of Practice: Learning, Meaning, and Identity. Cambridge University Press, Cambridge, New York (1998) 
8. Star, S.L.: This is not a boundary object: reflections on the origin of a concept. Sci. Technol. Hum. Values 35(5), 601-617 (2010)

9. Yakura, E.K.: Charting time: timelines as temporal boundary objects. Acad. Manag. J. 45(5), 956-970 (2002)

10. Briers, M., Chua, W.F.: The role of actor-networks and boundary objects in management accounting change: a field study of an implementation of activity-based costing. Acc. Organ. Soc. 26(3), 237-269 (2001)

11. Arias, E., et al.: Transcending the individual human mind - creating shared understanding through collaborative design. ACM Trans. Comput. Hum. Interact. 7, 84-113 (2000)

12. Sapsed, J., Salter, A.: Postcards from the edge: local communities, global programs and boundary objects. Organ. Stud. 25(9), 1515-1534 (2004)

13. Levina, N., Vaast, E.: The emergence of boundary spanning competence in practice: implications for implementation and use of information systems. MIS Quart. 29(2), 335-363 (2005)

14. Howard-Grenville, J.A., Carlile, P.R.: The incompatibility of knowledge regimes: consequences of the material world for cross-domain work. European J. Inf. Syst. 15(5), 473-485 (2006)

15. Carlile, P.R.: Transferring, translating, and transforming: an integrative framework for managing knowledge across boundaries. Organ. Sci. 15(5), 555-568 (2004)

16. Carlile, P.R.: A pragmatic view of knowledge and boundaries: boundary objects in new product development. Organ. Sci. 13(4), 442-455 (2002)

17. Akkerman, S.F., Bakker, A.: Boundary crossing and boundary objects. Rev. Educ. Res. 81 (2), 132-169 (2011)

18. Star, S.L.: This is not a boundary object: reflections on the origin of a concept. Sci. Technol. Hum. Values 35(5), 601-617 (2010)

19. Greenhalgh, T., Stones, R., Swinglehurst, D.: Choose and book: a sociological analysis of resistance to an expert system. SSM Soc. Sci. Med. 104, 210-219 (2014)

20. Peltu, M., Eason, K., Clegg, C.: How a Sociotechnical Approach Can Help NPfIT Deliver Better NHS Patient Care. BCS online, Sociotechnical Group (2008)

21. Schmidt, K.: Cooperative Work and Coordinative Practices Contributions to the Conceptual Foundations of Computer Supported Cooperative Work (CSCW). Springer, London, New York (2011)

22. Mingers, J., Willcocks, L.: An integrative semiotic framework for information systems: the social, personal and material worlds. Inf. Organ. 24, 48-70 (2014)

23. Edgley, A., et al.: Critical realist review: exploring the real, beyond the empirical. J. Furth. High. Educ. 40, 316-330 (2014)

24. Stamper, R.K.: Organisational semiotics: informatics without the computer? In: Liu, K., et al. (eds.) Information, Organisation and Technology: Studies in Organisational Semiotics, pp. 115-171. Springer, Boston (2001)

25. Deschamps, J.P., Nelson, B.: Innovation Governance: How Top Management Organizes and Mobilizes for Innovation. Wiley, Hoboken (2014) 